\title{
On a new fixed point theorem with an application on a coupled system of fractional differential equations
}

\author{
Hojjat Afshari' , Fahd Jarad ${ }^{2}$ and Thabet Abdeljawad ${ }^{3,4,5^{*}}$ (B)
}

\section{"Correspondence:}

tabdeljawad@psu.edu.sa

${ }^{3}$ Department of Mathematics and

General Sciences, Prince Sultan

University, P.O. Box 66833, 11586,

Riyadh, Saudi Arabia

${ }^{4}$ Department of Medical Research, China Medical University, 40402,

Taichung, Taiwan

Full list of author information is

available at the end of the article

\begin{abstract}
In this work, new theorems and results related to fixed point theory are presented. The results obtained are used for the sake of proving the existence and uniqueness of a positive solution of a coupled system of equations that involves fractional derivatives in the Riemann-Liouville settings and is subject to boundary conditions in the form of integrals.
\end{abstract}

Keywords: Fractional differential equation; Common fixed point; $\rho_{*}$-admissible; Coupled system

\section{Introduction}

For the last multiple of decades, the fractional calculus has drawn great interest of many sciences working on multifarious sciences on account of the good results obtained when this research applied the fractional tools to modeling of some real life problems [22, 26, $30,36,46-48]$.

On the other side, even though the fixed point theory is a branch of pure mathematics, it is showed that this theory is one of the main tools to use in order to tackle the qualitative properties of differential and integral equations in general and the existence and uniqueness of solutions to these equations in particular. A significant number of mathematicians utilized the classical results in the fixed point theory to discuss solutions of initial and boundary value problems (see [2-21, 23, 24, 27-29, 31-34, 37, 39-43]). Meanwhile, others established new fixed point theorems and applied them to proving the existence and oneness of solutions to a variety of differential equations [13, 25, 38, 44, 45].

In this work, we will propose new theorems related to the fixed points of operators. We discuss the admissibility of two multi-valued mappings in the category of complete b-metric spaces to obtain the existence of a common fixed point. Using the triangular admissibility, we prove the uniqueness of the common fixed point. Then we utilize the findings of this problem to discuss one of the most considerable qualitative aspects for differential equation with fractional order; that is, the existence and uniqueness of the positive solution of Riemann-Liouville fractional coupled system governed by boundary integral conditions.

(c) The Author(s) 2020. This article is licensed under a Creative Commons Attribution 4.0 International License, which permits use sharing, adaptation, distribution and reproduction in any medium or format, as long as you give appropriate credit to the original author(s) and the source, provide a link to the Creative Commons licence, and indicate if changes were made. The images or other third party material in this article are included in the article's Creative Commons licence, unless indicated otherwise in a credit line to the material. If material is not included in the article's Creative Commons licence and your intended use is not permitted by statutory regulation or exceeds the permitted use, you will need to obtain permission directly from the copyright holder. To view a copy of this licence, visit http://creativecommons.org/licenses/by/4.0/. 


\section{Preliminaries}

Let $\chi$ be a set of all increasing and continuous functions $\varphi:[0,+\infty) \rightarrow[0,+\infty)$ with the property $\varphi(\varsigma)=0$ if and only if $\varsigma=0$ and $\varphi(c \varsigma) \leq c \varphi(\varsigma)$ for $c>1$.

Let $\Omega$ be the family of all functions $\vartheta:[0,+\infty) \rightarrow\left[0, \frac{1}{s^{2}}\right)$ such that, for any bounded sequence $\left\{\zeta_{n}\right\}$ of positive real numbers, $\vartheta\left(\varsigma_{n}\right) \rightarrow 1$ implies $\varsigma_{n} \rightarrow 0$.

Let $(X, d)$ be a $b$-metric space. Take $C B(X)$ the set of bounded and closed sets in $X$. For $x \in X$ and $A, B \in C B(X)$, we define

$$
\begin{aligned}
& D(x, A)=\inf _{a \in A} d(x, a), \\
& D(A, B)=\sup _{a \in A} D(a, B) .
\end{aligned}
$$

Define a mapping $H: C B(X) \times C B(X) \rightarrow[0, \infty)$ such that

$$
H(A, B)=\max \left\{\sup _{x \in A} d(x, B), \sup _{y \in B} d(y, B)\right\}
$$

for every $A, B \in C B(X)$. Then the mapping $H$ forms a $b$-metric.

Throughout the article $I$ will denote the interval $[0,1]$. The definition for admissibility of a pair of single-valued mappings was first introduced in [1] and then generalized and used in [35] as well. Below we present the definition for the multi-valued case within the triangular admissibility.

Definition 2.1 Let $T_{1}, T_{2}: X \rightarrow C B(X)$ be two multi-valued mappings and $\rho: X \times X \rightarrow$ $[0,+\infty)$ be a function. Then the pair $\left(T_{1}, T_{2}\right)$ is said to be triangular $\rho_{*}$-admissible if the following conditions hold:

(i) $\left(T_{1}, T_{2}\right)$ is $\rho_{*}$-admissible; that is, $\rho(\varsigma, \eta) \geq 1$ implies $\rho_{*}\left(T_{1} \varsigma, T_{2} \eta\right) \geq 1$ and $\rho_{*}\left(T_{2} \varsigma, T_{1} \eta\right) \geq 1$, where

$$
\rho_{*}(A, B)=\inf \{\rho(\varsigma, \eta): \varsigma \in A, \eta \in B\}
$$

(ii) $\rho(\varsigma, u) \geq 1$ and $\rho(u, \eta) \geq 1$ imply $\rho(\varsigma, \eta) \geq 1$.

Definition 2.2 ([26, 36]) The Riemann-Liouville fractional integral of order $\rho>0$ of a continuous function $f:(0,+\infty) \rightarrow(-\infty,+\infty)$ is given by

$$
I_{0^{+}}^{\rho} f(\varsigma)=\frac{1}{\Gamma(\rho)} \int_{0}^{\varsigma}(\varsigma-\eta)^{\rho-1} f(\eta) d \eta
$$

provided the right-hand side is pointwise defined on $(0,+\infty)$.

Definition $2.3([26,36])$ The Riemann-Liouville fractional derivative of order $\rho>0$ of a continuous function $f:(0,+\infty) \rightarrow(-\infty,+\infty)$ is given by

$$
D_{0^{+}}^{\rho} f(\varsigma)=\frac{1}{\Gamma(n-\rho)}\left(\frac{d}{d t}\right)^{n} \int_{0}^{\varsigma}(\varsigma-\eta)^{n-\rho-1} f(\eta) d \eta,
$$

where $n=[\rho]+1,[\rho]$ denotes the integer part of the number $\rho$, provided that the righthand side is pointwise defined on $(0,+\infty)$. 
In this paper, we discuss the local existence and uniqueness of positive solutions for the following coupled system of fractional boundary value problem subject to integral boundary conditions:

$$
\left\{\begin{array}{l}
D_{0^{+}}^{\rho} u(\varsigma)+f(\varsigma, v(\varsigma))=0, \quad D_{0^{+}}^{\varrho} v(\varsigma)+g(\varsigma, u(\varsigma))=0, \quad 0<\varsigma<1, \\
u(0)=0, \quad u(1)=\int_{0}^{1} \phi(\varsigma) u(\varsigma) d \varsigma, \quad v(0)=0, \quad v(1)=\int_{0}^{1} \varphi(\varsigma) v(\varsigma) d \varsigma
\end{array}\right.
$$

where $1<\rho, \varrho \leq 2, \phi, \varphi \in L^{1} I$ are nonnegative and $f, g \in C(I \times[0,+\infty),[0,+\infty))$ and $D$ is the standard Riemann-Liouville fractional derivative. The functions $\phi(\varsigma), \varphi(\varsigma)$ satisfy the following conditions:

$$
(Q) \phi, \varphi: I \rightarrow[0,+\infty) \quad \text { with } \phi, \varphi \in L^{1} I
$$

and

$$
\begin{aligned}
& \sigma_{1}:=\int_{0}^{1} \phi(\varsigma) \varsigma^{\rho-1} d \varsigma, \quad \sigma_{2}:=\int_{0}^{1} \varphi(\varsigma) \varsigma^{\varrho-1} d \varsigma, \quad \varsigma \in(0,1) \\
& \sigma_{3}:=\int_{0}^{1} \varsigma^{\rho-1}(1-\varsigma) \phi(\varsigma) d \varsigma, \quad \sigma_{4}:=\int_{0}^{1} \varsigma^{\varrho-1}(1-\varsigma) \varphi(\varsigma) d \varsigma>0 .
\end{aligned}
$$

Lemma 2.4 ([41]) If $\int_{0}^{1} \phi(\varsigma) \varsigma^{\rho-1} d \varsigma \neq 1$, then, for any $\sigma \in C I$, the unique solution of the following boundary value problem:

$$
\left\{\begin{array}{l}
D_{0^{+}}^{\rho} u(\varsigma)+\sigma(\varsigma)=0, \quad 0<\varsigma<1 \\
u(0)=0, \quad u(1)=\int_{0}^{1} \phi(\varsigma) u(\varsigma) d \varsigma
\end{array}\right.
$$

is given by

$$
u(\varsigma)=\int_{0}^{1} G_{1 \rho}(\varsigma, \eta) \sigma(\eta) d \eta
$$

where

$$
\begin{aligned}
& G_{1 \rho}(\varsigma, \eta)=G_{2 \rho}(\varsigma, \eta)+G_{3 \rho}(\varsigma, \eta), \\
& G_{2 \rho}(\varsigma, \eta)=\frac{1}{\Gamma(\rho)} \begin{cases}\varsigma^{\rho-1}(1-\eta)^{\rho-1}-(\eta-\varsigma)^{\rho-1}, & 0 \leq \varsigma \leq \eta \leq 1, \\
\varsigma^{\rho-1}(1-\eta)^{\rho-1}, & 0 \leq \eta \leq \varsigma \leq 1,\end{cases} \\
& G_{3 \rho}(\varsigma, \eta)=\frac{\varsigma^{\rho-1}}{1-\int_{0}^{1} \phi(\varsigma) \varsigma^{\rho-1} d \varsigma} \int_{0}^{1} \phi(\varsigma) G_{2 \rho}(\varsigma, \eta) d \varsigma,
\end{aligned}
$$

Then $G(\varsigma, \eta)=\left(G_{1 \rho}(\varsigma, \eta), G_{1 \varrho}(\varsigma, \eta)\right)$ is a Green's function of the system $(1)$.

Lemma $2.5([41])$ Let $\rho, \varrho \in(1,2]$. Assume that $(Q)$ holds. Then the functions $G_{1 \rho}(\varsigma, \eta)$, $G_{1 \varrho}(\varsigma, \eta)$ have the following properties:

$$
\frac{(\rho-1) \sigma_{3} \eta(1-\eta)^{\rho-1} \varsigma^{\rho-1}}{\left(1-\sigma_{1}\right) \Gamma(\rho)} \leq G_{1 \rho} \leq \frac{(1-\eta)^{\rho-1} \varsigma^{\rho-1}}{\Gamma(\rho)\left(1-\sigma_{1}\right)}
$$




$$
\frac{(\varrho-1) \sigma_{4} \eta(1-\eta)^{\varrho-1} \varsigma^{\varrho-1}}{\left(1-\sigma_{2}\right) \Gamma(\varrho)} \leq G_{1 \varrho} \leq \frac{(1-\eta)^{\varrho-1} \varsigma^{\varrho-1}}{\Gamma(\varrho)\left(1-\sigma_{2}\right)}, \quad \eta, \varsigma \in I .
$$

Lemma 2.6 ([41]) Assume that $(Q)$ holds and $f(\kappa, x), g(\kappa, x)$ are continuous, then $(u, v) \in$ $X \times X$ is a solution of the system (1) if and only if it is a solution of the integral equations

$$
\left\{\begin{array}{l}
u(\kappa)=\int_{0}^{1} G_{1 \varrho}(\kappa, \varkappa) f(\varkappa, v(\varkappa)) d \varkappa, \\
v(\kappa)=\int_{0}^{1} G_{1 \rho}(\kappa, \varkappa) g(\varkappa, u(\varkappa)) d \varkappa .
\end{array}\right.
$$

\section{Main results}

Now, we are ready to state and prove our main results.

The following key lemma is essential to proceed in proving the main results. It states that the admissibility of a pair of multi-valued functions will guarantee the existence of a sequence of points with diameter greater than 1.

Lemma 3.1 Let $T_{1}, T_{2}: X \rightarrow C B(X)$ be two multi-valued mappings such that the pair $\left(T_{1}, T_{2}\right)$ is triangular $\rho_{*}$-admissible. Assume that there exists $\varrho_{0} \in X$ with $\rho_{*}\left(\varrho_{0}, T_{1} \varrho_{0}\right) \geq 1$. Define a sequence $\left\{\varrho_{n}\right\}$ in $X$ by $\varrho_{2 i+1} \in T_{1} \varrho_{2 i}$ and $\varrho_{2 i+2} \in T_{2} \varrho_{2 i+1}$, where $i=0,1,2, \ldots$. Then, for $m, n \in \mathbb{N} \cup\{0\}$ with $m>n$, we have $\rho\left(\varrho_{n}, \varrho_{m}\right) \geq 1$.

Proof From $\rho_{*}\left(\varrho_{0}, T_{1} \varrho_{0}\right) \geq 1$ we get $\rho\left(\varrho_{0}, \varrho_{1}\right) \geq 1$. Since $\left(T_{1}, T_{2}\right)$ is $\rho_{*}$-admissible, we obtain $\rho_{*}\left(T_{1} \varrho_{0}, T_{2} \varrho_{1}\right) \geq 1$, hence $\rho\left(\varrho_{1}, \varrho_{2}\right) \geq 1$ and so $\rho_{*}\left(T_{2} \varrho_{1}, T_{1} \varrho_{2}\right) \geq 1$, then $\rho\left(\varrho_{2}, \varrho_{3}\right) \geq 1$, with continuing this process we obtain, $\rho\left(\varrho_{m}, \varrho_{m+1}\right) \geq 1$.

By (ii) from definition of triangular $\rho_{*}$-admissible and regarding as; $\rho\left(\varrho_{n}, \varrho_{n+1}\right) \geq 1$ and $\rho\left(\varrho_{n+1}, \varrho_{n+2}\right) \geq 1$, deduce $\rho\left(\varrho_{n}, \varrho_{n+2}\right) \geq 1$. Again with continuing this process and from $m>n$, we find $\rho\left(\varrho_{n}, \varrho_{m}\right) \geq 1$.

The following theorem gives the existence of a common fixed point for two mappings $T_{1}$ and $T_{2}$ under less hypotheses than the results existing in the literature.

Theorem 3.2 Let $(X, d)$ be an $\rho$-complete b-metric space (with $s \geq 1$ ), and $\rho: X \times X \rightarrow$ $[0,+\infty)$ be a function. Suppose that $T_{1}, T_{2}: X \rightarrow C B(X)$ are mappings such that

$$
\rho(\varsigma, \eta) \varphi\left(s^{3} H\left(T_{1} \varsigma, T_{2} \eta\right)\right) \leq \vartheta(\varphi(M(\varsigma, \eta))) \varphi(M(\varsigma, \eta))+L \phi(N(\varsigma, \eta)),
$$

where

$$
\begin{aligned}
& M(\varsigma, \eta)=\max \left\{d(\varsigma, \eta), D\left(\varsigma, T_{1} \varsigma\right), D\left(\eta, T_{2} \eta\right), \frac{D\left(\eta, T_{1} \varsigma\right)+D\left(\varsigma, T_{2} \eta\right)}{2 s}\right\} \text { and } \\
& N(\varsigma, \eta)=\min \left\{D\left(\eta, T_{1} \varsigma\right), D\left(\eta, T_{2} \eta\right)\right\}
\end{aligned}
$$

for $\vartheta \in \Omega$ and $\varphi, \phi \in \chi$. Moreover, suppose

(i) $\left(T_{1}, T_{2}\right)$ is triangular $\rho_{*}$-admissible;

(ii) there exists $\varrho_{0} \in X$ with $\rho_{*}\left(\varrho_{0}, T_{1} \varrho_{0}\right) \geq 1$;

(iii) if for every sequence $\left\{\varrho_{n}\right\}$ in $X$ with $\rho\left(\varrho_{n}, \varrho_{n+1}\right) \geq 1$ for all $n \in \mathbb{N} \cup\{0\}$ and $\varrho_{n} \rightarrow \varrho \in X$, then there exists a subsequence $\left\{\varrho_{n(k)}\right\}$ of $\left\{\varrho_{n}\right\}$ with $\rho\left(\varrho_{n}(k), \varrho\right) \geq 1$.

Then $T_{1}$ and $T_{2}$ have a common fixed point $\varrho \in X$. 
Proof Let $\varrho_{0} \in X$ with $\rho_{*}\left(\varrho_{0}, T_{1} \varrho_{0}\right) \geq 1$. Choose $\varrho_{1} \in T_{1} \varrho_{0}$ such that $\rho\left(\varrho_{0}, \varrho_{1}\right) \geq 1$ and $\varrho_{1} \neq \varrho_{0}$. Let $q=\frac{1}{\sqrt{\vartheta\left(\varphi\left(d\left(\varrho_{0}, \varrho_{1}\right)\right)\right)}}$. By (3) and considering that $q>1$, we have

$$
0<\varphi\left(D\left(\varrho_{1}, T_{2} \varrho_{1}\right)\right) \leq \rho\left(\varrho_{0}, \varrho_{1}\right) \varphi\left(H\left(T_{1} \varrho_{0}, T_{2} \varrho_{1}\right)\right)<q \rho\left(\varrho_{0}, \varrho_{1}\right) \varphi\left(s^{3} H\left(T_{1} \varrho_{0}, T_{2} \varrho_{1}\right)\right) .
$$

Hence, there exists $\varrho_{2} \in T_{2} \varrho_{1}$ such that

$$
\begin{aligned}
\varphi\left(d\left(\varrho_{1}, \varrho_{2}\right)\right) & <q \rho\left(\varrho_{0}, \varrho_{1}\right) \varphi\left(s^{3} H\left(T_{1} \varrho_{0}, T_{2} \varrho_{1}\right)\right) \\
& \leq q \vartheta\left(\varphi\left(M\left(\varrho_{0}, \varrho_{1}\right)\right)\right) \varphi\left(M\left(\varrho_{0}, \varrho_{1}\right)\right)+q L \phi\left(N\left(\varrho_{0}, \varrho_{1}\right)\right) \\
& =\sqrt{\vartheta\left(\varphi\left(d\left(\varrho_{0}, \varrho_{1}\right)\right)\right)} \varphi\left(M\left(\varrho_{0}, \varrho_{1}\right)\right)+q L \phi\left(N\left(\varrho_{0}, \varrho_{1}\right)\right)
\end{aligned}
$$

where

$$
\begin{aligned}
M\left(\varrho_{0}, \varrho_{1}\right) & =\max \left\{d\left(\varrho_{0}, \varrho_{1}\right), D\left(\varrho_{0}, T_{1} \varrho_{0}\right), D\left(\varrho_{1}, T_{2} \varrho_{1}\right), \frac{D\left(\varrho_{1}, T_{1} \varrho_{0}\right)+D\left(\varrho_{0}, T_{2} \varrho_{1}\right)}{2 s}\right\} \\
& \leq \max \left\{d\left(\varrho_{0}, \varrho_{1}\right), D\left(\varrho_{1}, T_{2} \varrho_{1}\right), \frac{D\left(\varrho_{0}, T_{2} \varrho_{1}\right)}{2 s}\right\} \\
& \leq \max \left\{d\left(\varrho_{0}, \varrho_{1}\right), D\left(\varrho_{1}, T_{2} \varrho_{1}\right), \frac{D\left(\varrho_{0}, T_{2} \varrho_{1}\right)}{2 s}\right\}
\end{aligned}
$$

and

$$
\begin{aligned}
N\left(\varrho_{0}, \varrho_{1}\right) & =\min \left\{D\left(\varrho_{0}, T_{2} \varrho_{0}\right), D\left(\varrho_{1}, T_{1} \varrho_{0}\right)\right\} \\
& \leq \min \left\{d\left(\varrho_{0}, \varrho_{1}\right), d\left(\varrho_{1}, \varrho_{1}\right)\right\}=0 .
\end{aligned}
$$

Since

$$
\frac{D\left(\varrho_{0}, T_{2} \varrho_{1}\right)}{2} \leq \frac{\left[d\left(\varrho_{0}, \varrho_{1}\right)+D\left(\varrho_{1}, T_{2} \varrho_{1}\right)\right]}{2} \leq \max \left\{d\left(\varrho_{0}, \varrho_{1}\right), D\left(\varrho_{1}, T_{2} \varrho_{1}\right)\right\}
$$

we get

$$
M\left(\varrho_{0}, \varrho_{1}\right) \leq \max \left\{d\left(\varrho_{0}, \varrho_{1}\right), D\left(\varrho_{1}, T_{2} \varrho_{1}\right)\right\} .
$$

If $\max \left\{d\left(\varrho_{0}, \varrho_{1}\right), D\left(\varrho_{1}, T_{2} \varrho_{1}\right)\right\}=D\left(\varrho_{1}, T_{2} \varrho_{1}\right)$, then by (5), we have

$$
\varphi\left(D\left(\varrho_{1}, T_{2} \varrho_{1}\right)\right) \leq \varphi\left(d\left(\varrho_{1}, \varrho_{2}\right)\right)<\sqrt{\vartheta\left(\varphi\left(d\left(\varrho_{0}, \varrho_{1}\right)\right)\right)} \varphi\left(D\left(\varrho_{1}, T_{2} \varrho_{1}\right)\right)<\varphi\left(D\left(\varrho_{1}, T_{2} \varrho_{1}\right)\right),
$$

which is a contradiction. Hence, we obtain $\max \left\{d\left(\varrho_{0}, \varrho_{1}\right), D\left(\varrho_{1}, T_{2} \varrho_{1}\right)\right\}=d\left(\varrho_{0}, \varrho_{1}\right)$ and so by (5),

$$
\varphi\left(d\left(\varrho_{1}, \varrho_{2}\right)\right) \leq \sqrt{\vartheta\left(\varphi\left(d\left(\varrho_{0}, \varrho_{1}\right)\right)\right)} \varphi\left(d\left(\varrho_{0}, \varrho_{1}\right)\right) .
$$

Knowing that $\varphi \in \chi$ and regarding the fact that $\sqrt{\vartheta\left(\varphi\left(d\left(\varrho_{0}, \varrho_{1}\right)\right)\right)}<1$, we have

$$
\varphi\left(\frac{1}{\sqrt{\vartheta\left(\varphi\left(d\left(\varrho_{0}, \varrho_{1}\right)\right)\right)}} d\left(\varrho_{1}, \varrho_{2}\right)\right) \leq \frac{1}{\sqrt{\vartheta\left(\varphi\left(d\left(\varrho_{0}, \varrho_{1}\right)\right)\right)}} \varphi\left(d\left(\varrho_{1}, \varrho_{2}\right)\right)<\varphi\left(d\left(\varrho_{0}, \varrho_{1}\right)\right) .
$$


Since $\varphi$ is increasing,

$$
d\left(\varrho_{1}, \varrho_{2}\right) \leq \sqrt{\vartheta\left(\varphi\left(d\left(\varrho_{0}, \varrho_{1}\right)\right)\right)} d\left(\varrho_{0}, \varrho_{1}\right)
$$

Recall that $\varrho_{2} \in T_{2} \varrho_{1}$ and $\varrho_{1} \notin T_{2} \varrho_{1}$, so it is clear that $\varrho_{2} \neq \varrho_{1}$. Put

$$
q_{1}=\frac{\sqrt{\vartheta\left(\varphi\left(d\left(\varrho_{0}, \varrho_{1}\right)\right)\right)} \varphi\left(d\left(\varrho_{0}, \varrho_{1}\right)\right)}{\varphi\left(d\left(\varrho_{1}, \varrho_{2}\right)\right)} .
$$

By (6), we have $q_{1}>1$. Then

$$
0<\varphi\left(d\left(\varrho_{2}, T_{1} \varrho_{2}\right)\right) \leq \rho\left(\varrho_{1}, \varrho_{2}\right) \varphi\left(H\left(T_{2} \varrho_{1}, T_{1} \varrho_{2}\right)\right)<q_{1} \rho\left(\varrho_{1}, \varrho_{2}\right) \varphi\left(H\left(T_{2} \varrho_{1}, T_{1} \varrho_{2}\right)\right)
$$

Hence, there exists $\varrho_{3} \in T_{1} \varrho_{2}$ such that

$$
\begin{aligned}
\varphi\left(d\left(\varrho_{2}, \varrho_{3}\right)\right) & <q_{1} \rho\left(\varrho_{1}, \varrho_{2}\right) \varphi\left(H\left(T_{2} \varrho_{1}, T_{1} \varrho_{2}\right)\right) \\
& \leq q_{1} \vartheta\left(\varphi\left(M\left(\varrho_{1}, \varrho_{2}\right)\right)\right) \varphi\left(M\left(\varrho_{1}, \varrho_{2}\right)\right)+q_{1} L \phi\left(N\left(\varrho_{1}, \varrho_{2}\right)\right)
\end{aligned}
$$

Similarly, $M\left(\varrho_{1}, \varrho_{2}\right) \leq d\left(\varrho_{1}, \varrho_{2}\right)$ and $N\left(\varrho_{1}, \varrho_{2}\right)=0$. So by $(5)$ we have

$$
\begin{aligned}
\varphi\left(d\left(\varrho_{2}, \varrho_{3}\right)\right) & \leq \sqrt{\vartheta\left(\varphi\left(d\left(\varrho_{1}, \varrho_{2}\right)\right)\right)} \varphi\left(d\left(\varrho_{1}, \varrho_{2}\right)\right) \\
& \leq \sqrt{\vartheta\left(\varphi\left(d\left(\varrho_{1}, \varrho_{2}\right)\right)\right)} \sqrt{\vartheta\left(\varphi\left(d\left(\varrho_{1}, \varrho_{2}\right)\right)\right)} \varphi\left(d\left(\varrho_{0}, \varrho_{1}\right)\right) \\
& =\left(\sqrt{\left.\vartheta\left(\varphi\left(d\left(\varrho_{1}, \varrho_{2}\right)\right)\right)\right)^{2}} \varphi\left(d\left(\varrho_{0}, \varrho_{1}\right)\right) .\right.
\end{aligned}
$$

Again by (6), we obtain

$$
d\left(\varrho_{2}, \varrho_{3}\right) \leq\left(\sqrt{\vartheta\left(\varphi\left(d\left(\varrho_{1}, \varrho_{2}\right)\right)\right)}\right)^{2} d\left(\varrho_{0}, \varrho_{1}\right)
$$

It is clear that $\varrho_{2} \neq \varrho_{1}$. Put

$$
q_{2}=\frac{\left(\sqrt{\vartheta\left(\varphi\left(d\left(\varrho_{1}, \varrho_{2}\right)\right)\right)}\right)^{2} \varphi\left(d\left(\varrho_{0}, \varrho_{1}\right)\right)}{\varphi\left(d\left(\varrho_{2}, \varrho_{3}\right)\right)} .
$$

Then $q_{2}>1$ and we have

$$
0<\varphi\left(d\left(\varrho_{3}, T_{2} \varrho_{3}\right)\right) \leq \rho\left(\varrho_{2}, \varrho_{3}\right) \varphi\left(H\left(T_{1} \varrho_{2}, T_{2} \varrho_{3}\right)\right)<q_{2} \rho\left(\varrho_{2}, \varrho_{3}\right) \varphi\left(H\left(T_{1} \varrho_{2}, T_{2} \varrho_{3}\right)\right)
$$

Thus, there exists $\varrho_{4} \in T_{2} \varrho_{3}$ such that

$$
\begin{aligned}
\varphi\left(d\left(\varrho_{3}, \varrho_{4}\right)\right) & <q_{2} \rho\left(\varrho_{2}, \varrho_{3}\right) \varphi\left(H\left(T_{1} \varrho_{2}, T_{2} \varrho_{3}\right)\right) \\
& \leq q_{2} \vartheta\left(\varphi\left(M\left(\varrho_{2}, \varrho_{3}\right)\right)\right) \varphi\left(M\left(\varrho_{2}, \varrho_{3}\right)\right)+q_{2} L \phi\left(N\left(\varrho_{2}, \varrho_{3}\right)\right)
\end{aligned}
$$

Similarly, $M\left(\varrho_{2}, \varrho_{3}\right) \leq d\left(\varrho_{2}, \varrho_{3}\right)$ and $N\left(\varrho_{2}, \varrho_{3}\right)=0$. So by $(7)$,

$$
\varphi\left(d\left(\varrho_{3}, \varrho_{4}\right)\right) \leq \sqrt{\vartheta\left(\varphi\left(d\left(\varrho_{2}, \varrho_{3}\right)\right)\right)} \varphi\left(d\left(\varrho_{2}, \varrho_{3}\right)\right) \leq\left(\sqrt{\vartheta\left(\varphi\left(d\left(\varrho_{0}, \varrho_{1}\right)\right)\right)}\right)^{3} \varphi\left(d\left(\varrho_{0}, \varrho_{1}\right)\right) .
$$


Similarly, from (6), we obtain

$$
d\left(\varrho_{3}, \varrho_{4}\right) \leq\left(\sqrt{\vartheta\left(\varphi\left(d\left(\varrho_{0}, \varrho_{1}\right)\right)\right)}\right)^{3} d\left(\varrho_{0}, \varrho_{1}\right)
$$

It is clear that $\varrho_{3} \neq \varrho_{2}$. Put

$$
q_{3}=\frac{\left(\sqrt{\vartheta\left(\varphi\left(d\left(\varrho_{0}, \varrho_{1}\right)\right)\right)}\right)^{3} \varphi\left(d\left(\varrho_{0}, \varrho_{1}\right)\right)}{\varphi\left(d\left(\varrho_{2}, \varrho_{3}\right)\right)} .
$$

Then $q_{3}>1$. By continuing this process and by Lemma 3.1 we obtain a sequence $\left\{\varrho_{n}\right\}$ in $X$ such that

$$
\varrho_{2 i+1} \in T_{1} \varrho_{2 i} \quad \text { and } \quad \varrho_{2 i+2} \in T_{2} \varrho_{2 i+1}, \quad \text { where } i=0,1,2, \ldots
$$

Also, $d\left(\varrho_{n}, \varrho_{n+1}\right)<\left(\sqrt{\vartheta\left(\varphi\left(d\left(\varrho_{0}, \varrho_{1}\right)\right)\right)}\right)^{n} d\left(\varrho_{0}, \varrho_{1}\right)$ for all $n$. Let $t=\sqrt{\vartheta\left(\varphi\left(d\left(\varrho_{0}, \varrho_{1}\right)\right)\right)}$, then $0<$ $t<1$ for $n<m$, by the triangle inequality we have

$$
\begin{aligned}
d\left(\varrho_{n}, \varrho_{m}\right) \leq & d\left(\varrho_{n}, \varrho_{n+1}\right)+d\left(\varrho_{n+1}, \varrho_{n+2}\right)+\cdots \\
& +d\left(\varrho_{m-2}, \varrho_{m-1}\right)+d\left(\varrho_{m-1}, \varrho_{m}\right) \\
\leq & t^{n}\left(1+t+t^{2}+\cdots\right) d\left(\varrho_{0}, \varrho_{1}\right) \\
= & \left(\frac{t^{n}}{1-\varsigma}\right) d\left(\varrho_{0}, \varrho_{1}\right) \rightarrow 0 \text { as } n \rightarrow+\infty .
\end{aligned}
$$

Therefore, for $n<m$, we obtain

$$
d\left(\varrho_{n}, \varrho_{m}\right) \rightarrow 0 \quad \text { as } n \rightarrow+\infty
$$

Therefore

$$
\lim _{m, n \rightarrow \infty} d\left(\varrho_{n}, \varrho_{m}\right)=0
$$

We deduce that $\left\{\varrho_{n}\right\}$ is a Cauchy sequence in $(X, d)$. Since $(X, d)$ is a complete $b$-metric space, so there exists $\varrho^{*} \in X$ such that $\lim _{n \rightarrow \infty} \varrho_{n}=\varrho^{*}$. Since $\rho\left(\varrho_{n}, \varrho_{n+1}\right) \geq 1$, so there exists a subsequence $\left\{\varrho_{2 n_{k}}\right\}$ of $\left\{\varrho_{n}\right\}$ such that

$$
\rho\left(\varrho_{2 n_{k}}, \varrho^{*}\right) \geq 1
$$

for all $k$. By the triangular inequality

$$
\begin{aligned}
D\left(\varrho^{*}, T_{2} \varrho^{*}\right) & \leq s d\left(\varrho^{*}, \varrho_{2 n_{k}+1}\right)+s D\left(\varrho_{2 n_{k}+1}, T_{2} \varrho^{*}\right) \\
& \leq s d\left(\varrho^{*}, \varrho_{2 n_{k}+1}\right)+s H\left(T_{1} \varrho_{2 n_{k}}, T_{2} \varrho^{*}\right)
\end{aligned}
$$

Letting $k$ tend to infinity

$$
D\left(\varrho^{*}, T_{2} \varrho^{*}\right) \leq \lim _{k \rightarrow \infty} s H\left(T_{1} \varrho_{2 n_{k}}, T_{2} \varrho^{*}\right)
$$


Having $\varphi \in \chi,(8)$ and (9),

$$
\begin{aligned}
\varphi( & \left.s^{2} D\left(\varrho^{*}, T_{2} \varrho^{*}\right)\right) \\
& \leq \lim _{k \rightarrow \infty} \varphi\left(s^{3} H\left(T_{1} \varrho_{2 n_{k}}, T_{2} \varrho^{*}\right)\right) \\
& \leq \lim _{k \rightarrow \infty} \rho\left(\varrho_{2 n_{k}}, \varrho^{*}\right) \varphi\left(s^{3} H\left(T_{1} \varrho_{2 n_{k}}, T_{2} \varrho^{*}\right)\right) \\
& \leq \lim _{k \rightarrow \infty}\left[\vartheta\left(\varphi\left(M\left(\varrho_{2 n_{k}}, \varrho^{*}\right)\right)\right) \varphi\left(M\left(\varrho_{2 n_{k}}, \varrho^{*}\right)\right)+L \phi\left(N\left(\varrho_{2 n_{k}}, \varrho^{*}\right)\right)\right] .
\end{aligned}
$$

We have

$$
\begin{aligned}
& M\left(\varrho_{2 n_{k}}, \varrho^{*}\right) \\
& \quad=\max \left\{d\left(\varrho_{2 n_{k}}, \varrho^{*}\right), D\left(\varrho_{2 n_{k}}, T_{1} \varrho_{2 n_{k}}\right), D\left(\varrho^{*}, T_{2} \varrho^{*}\right), \frac{D\left(\varrho_{n_{k}}, T_{2} \varrho^{*}\right)+D\left(\varrho^{*}, T_{1} \varrho_{2 n_{k}}\right)}{2 s}\right\} \\
& \quad \leq \max \left\{d\left(\varrho_{2 n_{k}}, \varrho^{*}\right), d\left(\varrho_{2 n_{k}}, \varrho_{2 n_{k+1}}\right), D\left(\varrho^{*}, T_{2} \varrho^{*}\right), \frac{D\left(\varrho_{2 n_{k}}, T_{2} \varrho^{*}\right)+d\left(\varrho^{*}, \varrho_{2 n_{k}+1}\right)}{2 s}\right\}
\end{aligned}
$$

and

$$
\begin{aligned}
N\left(\varrho_{2 n_{k}}, \varrho^{*}\right) & =\min \left\{D\left(\varrho^{*}, T_{1} \varrho_{2 n_{k}}\right), D\left(\varrho^{*}, T_{2} \varrho^{*}\right)\right\} \\
& \leq \min \left\{d\left(\varrho^{*}, \varrho_{2 n_{k}+1}\right), d\left(\varrho^{*}, T_{2} \varrho^{*}\right)\right\} .
\end{aligned}
$$

Recall that

$$
\frac{D\left(\varrho_{2 n_{k}}, T_{2} \varrho^{*}\right)+d\left(\varrho^{*}, \varrho_{2 n_{k}+1}\right)}{2 s} \leq \frac{s d\left(\varrho_{2 n_{k}}, \varrho^{*}\right)+s D\left(\varrho^{*}, T_{2} \varrho^{*}\right)+d\left(\varrho^{*}, \varrho_{2 n_{k}+1}\right)}{2 s}
$$

Then

$$
\limsup _{k \rightarrow \infty} \frac{D\left(\varrho_{2 n_{k}}, T_{2} \varrho^{*}\right)+d\left(\varrho^{*}, \varrho_{2 n_{k}+1}\right)}{2 s} \leq \frac{D\left(\varrho^{*}, T_{2} \varrho^{*}\right)}{2}
$$

When $k$ tends to infinity, we deduce

$$
\lim _{k \rightarrow \infty} M\left(\varrho_{n_{k}}, \varrho^{*}\right)=D\left(\varrho^{*}, T_{2} \varrho^{*}\right)
$$

and

$$
\lim _{k \rightarrow \infty} N\left(\varrho_{n_{k}}, \varrho^{*}\right)=0
$$

Since $\lim _{k \rightarrow \infty} \varrho\left(\varphi\left(M\left(\varrho_{n_{k}}, \varrho^{*}\right)\right)\right) \leq \frac{1}{s^{2}}$, by $(10)$

$$
\varphi\left(s^{2} D\left(\varrho^{*}, T_{2} \varrho^{*}\right)\right) \leq \frac{1}{s^{2}} \varphi\left(D\left(\varrho^{*}, T_{2} \varrho^{*}\right)\right)
$$

Since $\varphi \in \chi$, the above holds unless $D\left(\varrho^{*}, T_{2} \varrho^{*}\right)=0$, that is, $\varrho^{*} \in T_{2} \varrho^{*}$. Similarly, we can prove that $\varrho^{*} \in T_{1} \varrho^{*}$, so $\varrho^{*}$ is a common fixed point of $T_{2}$ and $T_{1}$. 
Corollary 3.3 Let $(X, d)$ be an $\rho$-complete b-metric space, and $\rho: X \times X \rightarrow[0,+\infty)$ be a function. Suppose that $T_{1}, T_{2}: X \rightarrow X$ are mappings such that

$$
\rho(\varsigma, \eta) \varphi\left(s^{3} H\left(T_{1} \varsigma, T_{2} \eta\right)\right) \leq \vartheta(\varphi(d(\varsigma, \eta))) \varphi(d(\varsigma, \eta))
$$

for $\varrho \in \Omega$ and $\varphi \in \chi$. Moreover, suppose

(i) $\left(T_{1}, T_{2}\right)$ is triangular $\rho_{*}$-admissible;

(ii) there exists $\varrho_{0} \in X$ with $\rho\left(\varrho_{0}, T_{1} \varrho_{0}\right) \geq 1$;

(iii) if for every sequence $\left\{\varrho_{n}\right\}$ in $X$ with $\rho\left(\varrho_{n}, \varrho_{n+1}\right) \geq 1$ for all $n \in \mathbb{N} \cup\{0\}$ and

$\varrho_{n} \rightarrow \varrho \in X$, then there exists a subsequence $\left\{\varrho_{n(k)}\right\}$ of $\left\{\varrho_{n}\right\}$ with $\rho\left(\varrho_{n(k)}, \varrho\right) \geq 1$ for all $k$.

Then $T_{1}$ and $T_{2}$ have a common fixed point $\varrho \in X$.

Moreover, if the following condition holds:

$H_{1}$ : Either $\rho(u, v) \geq 1$ or $\rho(v, u) \geq 1$ whenever $T_{1} u=T_{2} u=u$ and $T_{1} v=T_{2} v=v$, then $T_{1}$ and $T_{2}$ have a unique common fixed point.

Proof The proof of the existence of a common fixed point of $T_{1}$ and $T_{2}$ was shown in Theorem 3.2.

We claim that, if $T_{1} u=T_{2} u=u$ and $T_{1} v=T_{2} v=v$, then $u=v$. By hypothesis, if $u \neq v$, then either $\rho(u, v) \geq 1$ or $\rho(v, u) \geq 1$. Suppose that $\rho(u, v) \geq 1$, then

$$
\begin{aligned}
\psi(d(u, v)) & =\psi(d(S u, T v)) \leq \psi\left(s^{3} d(S u, T v)\right) \leq \rho(u, v) \psi\left(s^{3} d(S u, T v)\right) \\
& \leq \vartheta(\psi(d(u, v))) \psi(d(u, v))<\psi(d(u, v)),
\end{aligned}
$$

which is contradiction. So $u=v$. Similarly, if $\rho(v, u) \geq 1$, we can prove $u=v$.

Theorem 3.4 Suppose for $\kappa \in I$ and $\eta, z \in C(I)$ there exists $\xi: \mathbb{R}^{2} \rightarrow \mathbb{R}$ and $\varphi \in \chi$ such that

(i)

$$
|g(\kappa, \eta(\kappa))-f(\kappa, z(\kappa))| \leq \frac{1}{2 \sqrt{2}} \frac{\Gamma(\max \{\rho, \varrho\})}{(1-\varkappa)^{\min \{\rho, \varrho\}-1} \kappa^{\min \{\rho, \varrho\}-1}} \frac{\varphi\left(|(\kappa)-z(\kappa)|^{2}\right)}{\sqrt{4\left\|(\eta-z)^{2}\right\|_{\infty}+1}}
$$

(ii) $\exists \eta_{0} \in C(I)$ with $\xi\left(\eta_{0}(\kappa), \int_{0}^{1} G(\kappa, \varkappa) f\left(\varkappa, \eta_{0}(\varkappa)\right) d \varkappa \geq 0\right.$.

If we set

$$
\begin{aligned}
& \eta_{1}=T_{1} \eta_{0}=\int_{0}^{1} G_{1 \rho}(\kappa, \varkappa) g\left(\varkappa, \eta_{0}(\varkappa)\right) d \varkappa ; \\
& \eta_{2}=T_{2} \eta_{1}=\int_{0}^{1} G_{1 \varrho}(\kappa, \varkappa) f\left(\varkappa, \eta_{1}(\varkappa)\right) d \varkappa ; \\
& \eta_{3}=T_{1} \eta_{2}=\int_{0}^{1} G_{1 \rho}(\kappa, \varkappa) g\left(\varkappa, \eta_{2}(\varkappa)\right) d \varkappa ; \\
& \vdots \\
& \eta_{2 n}=T_{2} \eta_{2 n-1}=\int_{0}^{1} G_{1 \varrho}(\kappa, \varkappa) f\left(\varkappa, \eta_{2 n-1}(\varkappa)\right) d \varkappa ;
\end{aligned}
$$




$$
\eta_{2 n+1}=T_{1} \eta_{2 n}=\int_{0}^{1} G_{1 \rho}(\kappa, \varkappa) g\left(\varkappa, \eta_{2 n}(\varkappa)\right) d \varkappa
$$

we may assume the following conditions are met:

(iii) $\xi\left(\eta_{2 n-1}, \eta_{2 n}\right) \geq 0$ and $\xi\left(\eta_{2 n}, \eta_{2 n+1}\right) \geq 0$, respectively, imply $\xi\left(\eta_{2 n}, \eta_{2 n+1}\right) \geq 0$ and $\xi\left(\eta_{2 n+1}, \eta_{2 n+2}\right) \geq 0$, respectively;

(iv) $\xi\left(\eta_{2 n-1}, \eta_{2 n}\right) \geq 0$ and $\xi\left(\eta_{2 n}, \eta_{2 n+1}\right) \geq 0$ implies $\xi\left(\eta_{2 n-1}, \eta_{2 n+1}\right) \geq 0$;

(v) iffor every sequence $\left\{\eta_{n}\right\}$ in $X$ with $\xi\left(\eta_{n}, \eta_{n+1}\right) \geq 0$ for all $n \in \mathbb{N} \cup\{0\}$ and $\eta_{n} \rightarrow \eta \in X$, then there exists a subsequence $\left\{\eta_{n(k)}\right\}$ of $\left\{\eta_{n}\right\}$ with $\xi\left(\eta_{n(k)}, \eta\right) \geq 1$ for all $k$.

Then the system (1) has a solution in $C(I)$.

Moreover, if the following conditions hold:

$H_{2}$ : Either $\xi\left(\eta^{*}, \zeta^{*}\right) \geq 0$ or $\xi\left(\zeta^{*}, \eta^{*}\right) \geq 0$ whenever

$$
\int_{0}^{1} G_{1 \rho}(\kappa, \varkappa) g\left(\varkappa, \eta^{*}(\varkappa)\right) d \varkappa=\int_{0}^{1} G_{1 \varrho}(\kappa, \varkappa) f\left(\varkappa, \eta^{*}(\varkappa)\right) d \varkappa=\eta^{*}
$$

and

$$
\int_{0}^{1} G_{1 \rho}(\kappa, \varkappa) g\left(\varkappa, \zeta^{*}(\varkappa)\right) d \varkappa=\int_{0}^{1} G_{1 \varrho}(\kappa, \varkappa) f\left(\varkappa, \zeta^{*}(\varkappa)\right) d \varkappa \zeta^{*}=\zeta^{*}
$$

then the system (1) has a unique solution in $C(I)$.

Proof By Lemma $2.6 \eta \in C(I)$ is a solution of (1) if and only if it is a solution of

$$
\left\{\begin{array}{l}
z=\int_{0}^{1} G_{1 \varrho}(\kappa, \varkappa) f(\varkappa, \eta(\varkappa)) d \varkappa \\
\eta=\int_{0}^{1} G_{1 \rho}(\kappa, \varkappa) g(\varkappa, z(\varkappa)) d \varkappa .
\end{array}\right.
$$

We define $T_{1}, T_{2}: C(I) \rightarrow C(I)$ by

$$
\begin{aligned}
& T_{1} \eta_{2 n}=\int_{0}^{1} G_{1 \rho}(\kappa, \varkappa) g\left(\varkappa, \eta_{2 n}(\varkappa)\right) d \varkappa, \\
& T_{2} \eta_{2 n-1}=\int_{0}^{1} G_{1 \varrho}(\kappa, \varkappa) f\left(\varkappa, \eta_{2 n-1}(\varkappa)\right) d \varkappa,
\end{aligned}
$$

for all $\kappa \in I$. For this purpose, we find a common fixed point of $T_{1}$ and $T_{2}$. Let $\eta, z \in C(I)$ with $\xi(\eta(\kappa), z(\kappa)) \geq 0$ for all $\kappa \in I$. By using (i), we get

$$
\begin{aligned}
\left|T_{1} \eta(\kappa)-T_{2} z(\kappa)\right|^{2} & =\left|\int_{0}^{1} G_{1 \rho}(\kappa, \varkappa)\left(g(\varkappa, \eta(\varkappa))-\int_{0}^{1} G_{1 \varrho}(\kappa, \varkappa) f(\varkappa, z(\varkappa))\right) d \varkappa\right|^{2} \\
& \leq\left[\int_{0}^{1} \frac{(1-\varkappa)^{\min \{\rho, \varrho\}-1} \kappa^{\min \{\rho, \varrho\}-1}}{\Gamma(\max \{\rho, \varrho\})}|h(\varkappa, \eta(\varkappa))-h(\varkappa, z(\varkappa))| d \varkappa\right]^{2} \\
& \leq\left[\int_{0}^{1} \frac{1}{2 \sqrt{2}} \frac{\varphi\left(|\eta(\varkappa)-z(\varkappa)|^{2}\right)}{\sqrt{4\left\|(\eta-z)^{2}\right\|_{\infty}+1}} d \varkappa\right]^{2}
\end{aligned}
$$




$$
\leq \frac{1}{8} \frac{\left(\varphi\left(\left\|(\eta-z)^{2}\right\|_{\infty}\right)\right)^{2}}{4\left\|(\eta-z)^{2}\right\|_{\infty}+1}
$$

Hence, for $\eta, z \in C(I), \kappa \in I$ with $\xi(\eta(\kappa), z(\kappa)) \geq 0$, we have

$$
\left\|\left(T_{1} \eta-T_{2} z\right)^{2}\right\|_{\infty} \leq \frac{1}{8} \frac{\left(\varphi\left(\left\|(\eta-z)^{2}\right\|_{\infty}\right)\right)^{2}}{4\left\|(\eta-z)^{2}\right\|_{\infty}+1} .
$$

Put $\rho: C(I) \times C(I) \rightarrow[0,+\infty)$ by

$$
\rho(\eta, z)= \begin{cases}1 & \xi(\eta(\kappa), z(\kappa)) \geq 0, \text { for all } \kappa \in I, \\ 0 & \text { else }\end{cases}
$$

Setting $\vartheta:[0,+\infty) \rightarrow\left[0, \frac{1}{4}\right)$ with $\vartheta(q)=\frac{q}{4 q+1}$ and $s=2$ we can obtain

$$
\begin{aligned}
\rho(\eta, z) \varphi\left(8 d\left(T_{1} \eta, T_{2} z\right)\right) & \leq 8 \rho(\eta, z) \varphi\left(d\left(T_{1} \eta, T_{2} z\right)\right) \\
& \leq \frac{(\varphi(d(\eta, z)))^{2}}{4 d(\eta, z)+1} \\
& \leq \frac{(\varphi(d(\eta, z)))^{2}}{4 \varphi(d(\eta, z))+1} \\
& =\frac{1}{\vartheta(\varphi(d(\eta, z)))} \vartheta(\varphi(d(\eta, z))) \frac{(\varphi(d(\eta, z)))^{2}}{4 \varphi(d(\eta, z))+1} \\
& \leq \vartheta(\varphi(d(\eta, z))) \varphi(d(\eta, z)), \quad \vartheta \in \Omega .
\end{aligned}
$$

So $T_{1}$ and $T_{2}$ obey all the conditions of Theorem 3.2. We find $\eta^{*} \in C(I)$ with $\eta^{*}=T_{1} \eta^{*}=$ $T_{2} \eta^{*}$.

Now we claim that the solution of coupled system (1) is unique. By the condition $H_{2}$ we have the following.

Either $\rho\left(\eta^{*}, \zeta^{*}\right) \geq 0$ or $\rho\left(\zeta^{*}, \eta^{*}\right) \geq 0$ whenever

$$
T_{1} \eta^{*}=T_{2} \eta^{*}=\eta^{*}
$$

and

$$
T_{1} \zeta^{*}=T_{2} \zeta^{*}=\zeta^{*}
$$

Now, using the condition of $H_{1}$ in Corollary 3.3, we obtain $\eta^{*}=\zeta^{*}$

\section{Conclusion}

Fixed point theory is one of the main tools of pure mathematics that are used to serve in the development in the qualitative theory of differential and integral equations. For the last few years, researchers have not only used the traditional fixed point theorems in proving the existence and uniqueness of solutions to various types of fractional differential equation, but have developed new fixed point theorems and applied them as well. In this article, we have developed a new fixed point theorem and utilized it to prove the local existence of positive solution to a coupled system of differential equations where the 
Riemann-Liouville fractional derivative is used. We believe that the new fixed point theorem considered here can be used to handle fractional differential equations in the setting of other derivatives, not only containing singular kernels, but nonsingular kernels as well.

\section{Acknowledgements}

The third author would like to thank Prince Sultan University for funding this work through research group Nonlinear Analysis Methods in Applied Mathematics (NAMAM) group number RG-DES-2017-01-17.

\section{Funding}

Not applicable.

\section{Availability of data and materials}

Data sharing not applicable to this article as no data sets were generated or analysed during the current study.

\section{Competing interests}

The authors declare that they have no competing interests.

\section{Authors' contributions}

All authors equally contributed in this manuscript and approved the final version.

\section{Author details}

'Department of Mathematics, Faculty of Basic Science, University of Bonab, Bonab, Iran. ${ }^{2}$ Department of Mathematics, Çankaya University, 06790, Ankara, Etimesgut, Turkey. ${ }^{3}$ Department of Mathematics and General Sciences, Prince Sultan University, P.O. Box 66833, 11586, Riyadh, Saudi Arabia. ${ }^{4}$ Department of Medical Research, China Medical University, 40402, Taichung, Taiwan. ${ }^{5}$ Department of Computer Science and Information Engineering, Asia University, Taichung, Taiwan.

\section{Publisher's Note}

Springer Nature remains neutral with regard to jurisdictional claims in published maps and institutional affiliations.

Received: 12 May 2020 Accepted: 24 August 2020 Published online: 03 September 2020

\section{References}

1. Abdeljawad, T.: Meir-Keeler $\alpha$-contractive fixed and common fixed point theorems. Fixed Point Theory Appl. 2013, 19 (2013)

2. Afshari, H.: Solution of fractional differential equations in quasi-b-metric and b-metric-like spaces. Adv. Differ. Equ. 2018, 285 (2018). https://doi.org/10.1186/s13662-019-2227-9

3. Afshari, H., Alsulami, H.H., Karapinar, E.: On the extended multivalued Geraghty type contractions. J. Nonlinear Sci. Appl. 9, 4695-4706 (2016). https://doi.org/10.22436/jnsa.009.06.108

4. Afshari, H., Baleanu, D.: Applications of some fixed point theorems for fractional differential equations with Mittag-Leffler kernel. Adv. Differ. Equ. 2020, 140 (2020). https://doi.org/10.1186/s13662-020-02592-2

5. Afshari, H., Kalantari, S., Baleanu, D.: Solution of fractional differential equations via $\alpha-\psi$-Geraghty type mappings. Adv. Differ. Equ. 2018, 347 (2018). https://doi.org/10.1186/s13662-018-1807-4

6. Afshari, H., Kalantari, S., Karapinar, E.: Solution of fractional differential equations via coupled fixed point. Electron. J. Differ. Equ. 2015(286), 1 (2015)

7. Afshari, H., Rezapour, S., Shahzad, N.: Absolute retract of of the common fixed points set of two multi-functions. Topol. Methods Nonlinear Anal. 40, 429-436 (2012)

8. Afshari, H., Sajjadmanesh, M., Baleanu, D.: Existence and uniqueness of positive solutions for a new class of coupled system via fractional derivatives. Adv. Differ. Equ. 2020(111), 1 (2020). https://doi.org/10.1186/s13662-020-02568-2

9. Ahmad, B., Nieto, J.: Existence results for a coupled system of nonlinear fractional differential equations with three-point boundary conditions. Comput. Math. Appl. 58, 1838-1843 (2009)

10. Ahmad, B., Ntouyas, S.K., Alsaedi, A.: On a coupled system of fractional differential equations with coupled nonlocal and integral boundary conditions. Chaos Solitons Fractals 83, 234-241 (2016)

11. Aleksić, S., Mitrović, Z.D., Radenović, S.: Picard sequences in b-metric spaces. Fixed Point Theory 21(1), 35-46 (2020)

12. Ali, A., Rabiei, F., Shah, K.: On Ulam's type stability for a class of impulsive fractional differential equations with nonlinear integral boundary conditions. J. Nonlinear Sci. Appl. 10, 4760-4775 (2017) https://doi.org/10.22436/jnsa.010.09.19

13. Alqahtani, B., Fulga, A., Jarad, F., Karapinar, E.: Nonlinear F-contractions on b-metric spaces and differential equations in the frame of fractional derivatives with Mittag-Leffler kernel. Chaos Solitons Fractals 128, 349-354 (2019)

14. Arshad, A., Shah, K., Jarad, F.: Ulam-Hyers stability analysis to a class of nonlinear implicit impulsive fractional differential equations with three point boundary conditions. Adv. Differ. Equ. 2019(7), 1 (2019). https://doi.org/10.1186/s13662-018-1943-x

15. Arshad, A., Shah, K., Jarad, F., Gupta, V., Abdeljawad, T.: Existence and stability analysis to a coupled system of implicit type impulsive boundary value problems of fractional-order differential equations. Adv. Differ. Equ. 2019(101), 1 (2019). https://doi.org/10.1186/s13662-019-2047-y

16. Bai, C., Fang, J.: The existence of a positive solution for a singular coupled system of nonlinear fractional differential equations. Appl. Math. Comput. 150, 611-621 (2004)

17. Bai, Z., Lü, H.: Positive solutions for boundary value problem of nonlinear fractional differential equation. J. Math. Anal. Appl. 311(2), 495-505 (2005) 
18. Daftardar-Gejji, V: Positive solutions of a system of non-autonomous factional differential equations. J. Math. Anal. Appl. 302, 56-64 (2005)

19. Feng, H., Zhai, C.: Existence and uniqueness of positive solutions for a class of fractional differential equation with integral boundary conditions. Nonlinear Anal., Model. Control 22(2), 160-172 (2017)

20. Goodrich, C.S.: Existence of a positive solution to a class of fractional differential equations. Appl. Math. Lett. 23(9), 1050-1055 (2010)

21. Goodrich, C.S.: Existence of a positive solution to systems of differential equations of fractional order. Comput. Math. Appl. 62(3), 1251-1268 (2011)

22. Hilfer, R.: Applications of Fractional Calculus in Physics. Word Scientific, Singapore (2000)

23. Infante, G., Pietramala, P.: Multiple nonnegative solutions of systems with coupled nonlinear boundary conditions. Math. Methods Appl. Sci. 37, 2080-2090 (2014)

24. Jeli, M., Samet, B.: Existence of positive solutions to an arbitrary order fractional differential equation via a mixed monotone operator method. Nonlinear Anal., Model. Control 20(3), 367-376 (2015)

25. Karapinar, E., Abdeljawad, T., Jarad, F.: Applying new fixed point theorems on fractional and ordinary differential equations. Adv. Differ. Equ. 2019, 421 (2019)

26. Kilbas, A.A., Srivastava, H.M., Trujillo, J.J.: Theory and Applications of Fractional Differential Equations. North-Holland Mathematics Studies, vol. 204 (2006)

27. Kukić, K., Shatanawi, W., Gardaöević-Filipović, M.: Khan and Ćirić contraction principles in almost b-metric spaces. UPB Sci. Bull., Ser. A 82(1) (2020)

28. Liu, L.L., Zhang, X.Q., Liu, L.S., Wu, Y.H.: Iterative positive solutions for singular nonlinear fractional differential equation with integral boundary conditions. Adv. Differ. Equ. 2016(1), 154 (2016)

29. L, C.: Some Recent Results in Metrical Fixed Point Theory. University of Belgrade, Beograd (2003)

30. Magin, R.L.: Fractional Calculus in Bioengineering. Begell House Publishers, (2006)

31. Malkowski, E., Rakočević, V.: Advanced Functional Analysis. CRS Press, Boca Raton (2019)

32. Marasi, H.R., Afshari, H., Zhai, C.B.: Some existence and uniqueness results for nonlinear fractional partial differential equations. Rocky Mt. J. Math. 47, 571-585 (2017). https://doi.org/10.1216/RMJ-2017-47-2-1

33. Mitrović, Z.D.: On an open problem in rectangular b-metric space. J. Anal. 25, 135-137 (2017)

34. Mitrović, Z.D.: A note on the result of Suzuki, Miculescu and Mihail. J. Fixed Point Theory Appl. 21, 24 (2019). https://doi.org/10.1007/s11784-019-0663-5

35. Patel, D.K., Abdeljawad, T., Gopal, D.: Common fixed points of generalized Meir-Keeler $\alpha$-contractions. Fixed Point Theory Appl. 2013, 260 (2013)

36. Podlubny, l.: Fractional Differential Equations. Academic Press, New york (1999)

37. Shah, K., Ali, A., Bushnaq, S.: Hyers-Ulam stability analysis to implicit Cauchy problem of fractional differential equations with impulsive conditions. Math. Methods Appl. Sci., 1-15 (2018). https://doi.org/10.1002/mma.5292

38. Shaoib, M., Abdeljawad, T., Sarwar, M., Jarad, F.: Fixed point theorems for multi-valued contractions in metric spaces with applications to fractional differential and integral equations. IEEE Access 7, 127373-127383 (2019)

39. Sun, Y., Zhao, M.: Positive solutions for a class of fractional differential equations with integral boundary conditions. Appl. Math. Lett. 34, 17-21 (2014)

40. Todorčević, V.: Harmonic Quasiconformal Mappings and Hyperbolic Type Metrics. Springer, Switzerland (2019)

41. Yang, C., Zhai, C., Zhang, L.: Local uniqueness of positive solutions for a coupled system of fractional differential equations with integral boundary conditions. Adv. Differ. Equ. 2017, 282 (2017)

42. Yang, W: Positive solutions for a coupled system of nonlinear fractional differential equations with integral boundary conditions. Comput. Math. Appl. 63(1), 288-297 (2012)

43. Yuan, C.: Two positive solutions for $(n-1,1)$-type semigroup integral boundary value problems for coupled systems of nonlinear fractional differential equations. Commun. Nonlinear Sci. Numer. Simul. 18, 858-866 (2013)

44. Zhai, C.B., Yan, W.P., Yang, C.: A sum operator method for the existence and uniqueness of positive solutions to Riemann-Liouville fractional differential equation boundary value problems. Commun. Nonlinear Sci. Numer. Simul. $18,858-866(2013)$

45. Zhai, C.B., Zhang, L.L.: New fixed point theorems for a mixed monotone operators and local existence-uniqueness of positive solutions for nonlinear boundary value problems. J. Math. Anal. Appl. 382, 594-614 (2011)

46. Zhou, B., Zhang, L.: Existence of positive solutions of boundary value problems for high-order nonlinear conformable differential equations with $p$-Laplacian operator. Adv. Differ. Equ. 2019, 351 (2019)

47. Zhou, B., Zhang, L., Addai, E., Zhang, N.: Multiple positive solutions for nonlinear high-order Riemann-Liouville fractional differential equations boundary value problems with $p$-Laplacian operator. Bound. Value Probl. 2020, 26 (2020)

48. Zhou, B., Zhang, L., Zhang, N., Addai, E.: Existence and monotone iteration of unique solution for tempered fractional differential equations Riemann-Stieltjes integral boundary value problems. Adv. Differ. Equ. 2020, 208 (2020) 